ACS Chem Biol. 2016 November 18; 11(11): 3106-3113. doi:10.1021/acschembio.6b00692.

\title{
Structural Aspects of Heparan Sulfate Binding to Robo1-lg1-2
}

\author{
Qi Gao, Cheng-Yu Chen, Chengli Zong, Shuo Wang, Annapoorani Ramiah, Pradeep \\ Prabhakar, Laura C. Morris, Geert-Jan Boons, Kelley W. Moremen, and James H. \\ Prestegard ${ }^{*}$ \\ Complex Carbohydrate Research Center, University of Georgia, Athens, Georgia 30602, United \\ States
}

\begin{abstract}
Roundabout 1, or Robo1, is a cell surface signaling molecule important in axon guidance. Its interaction with heparan sulfate (HS) and members of the Slit protein family is essential to its activity, making characterization of these interactions by structural methods, such as NMR, highly desirable. However, the fact that Robo1 is a glycosylated protein prevents employment of commonly used bacterial hosts for expression of properly glycosylated forms with the uniform ${ }^{15} \mathrm{~N},{ }^{13} \mathrm{C}$, and ${ }^{2} \mathrm{H}$ labeling needed for NMR studies. Here, we apply an alternative methodology, based on labeling with a single amino acid type and high structural content NMR data, to characterize a two-domain construct of glycosylated Robo1 (Robo1-Ig1-2) interacting with a synthetic HS tetramer (IdoA-GlcNS6S-IdoA2S-GlcNS6S- $\left.\left(\mathrm{CH}_{2}\right)_{5} \mathrm{NH}_{2}\right)$. Significant chemical shift perturbations of the crosspeak from K81 on titration with the tetramer provide initial evidence for the location of a binding site and allow determination of a $255 \mu \mathrm{M}$ disassociation constant. The binding epitopes, bound conformation, and binding site placement of the HS tetramer have been further characterized by saturation transfer difference (STD), transferred nuclear Overhauser effect (trNOE), and paramagnetic perturbation experiments. A model of the complex has been generated using constraints derived from the various NMR experiments. Postprocessing energetic analysis of this model provides a rationale for the role each glycan residue plays in the binding event, and examination of the binding site in the context of a previous Robo-Slit structure provides a rationale for modulation of Robo-Slit interactions by HS.
\end{abstract}

\section{Graphical abstract}

*Corresponding Author: jpresteg@ccrc.uga.edu.

Notes: The authors declare no competing financial interest.

Supporting Information: The Supporting Information is available free of charge on the ACS Publications website at DOI: 10.1021/ acschem-bio.6b00692. 


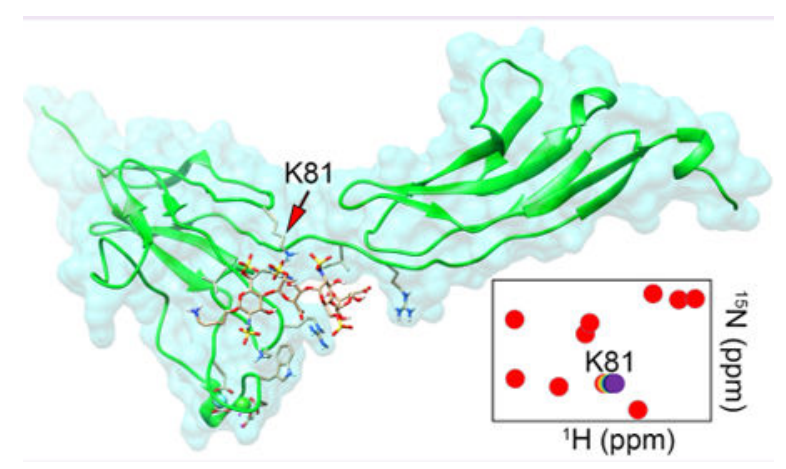

Robo1 (roundabout receptor 1) is one of four members of the human Robo family; all are developmentally important cell-surface signaling molecules recognized for their role in axon guidance, ${ }^{1}$ angiogenesis ${ }^{2}$ and the development of many internal organs. ${ }^{3,4}$ Changes in Robo1 levels are also correlated with tumorigenesis, cancer progression, and metastasis. ${ }^{5}$ Robo1 signaling is regulated by interactions with Slit2, one of three members of a family of very large secreted glycoproteins. Interactions between Robos and Slits are further modulated by interactions with heparn sulfate (HS; ${ }^{1,6}$ Figure $\left.1 \mathrm{~A}\right)$. There is evidence that the interactions with HS vary depending on particular sulfation patterns and other structural characteristics of this polymeric ligand. ${ }^{7}$ Producing a structure illustrating specific interactions between protein and ligand for a well-defined HS oligomer would provide a basis for understanding this specificity and using it in the design of molecules that could compete in modulating important physiological processes. Here, we present a model for the interaction of a particular HS tetramer (IdoA-GlcNS6S-IdoA2S-GlcNS6S- $\left(\mathrm{CH}_{2}\right)_{5} \mathrm{NH}_{2}$,

Figure 1B) with the terminal two domains of Robo1. The model is based on a combination of NMR cross-relaxation data that define bound ligand geometry, saturation transfer difference (STD) data that identify binding epitopes of the ligand, and perturbations of protein chemical shifts by the ligand, or ligand chemical shifts by a lanthanide binding tag on the protein, that locate the binding site. Docking a more extended octamer into the identified Robo1 binding site in a model of the Robo1- Slit2 complex suggests that HS interactions with Slit2 could modulate function.

Robo1 is a glycoprotein with an extracellular $\mathrm{N}$ terminal domain composed of five Ig motifs and two fibronectin motifs, a single trans-membrane helix, and a cytosolic domain. ${ }^{8}$ The two terminal domains (Ig1-2), which we have selected for this study, are believed to be directly involved with Slit and HS interactions. There is a substantial amount of previous structural information, including crystal structures of two domain constructs from both human and Drosophila homologues 9,10 that provide a useful starting point for our studies. However, questions remain about several structural aspects. Relative domain orientations appear to be quite variable, as evidenced by the differences in domain orientations among different crystal structures. Also, the heparin fragment found between two Robo1-Ig1-2 constructs in one crystal structure suggests dimerization may play a role, but there is no evidence for dimerization when binding is studied in solution. Moreover, the previous structures have used nonglycosylated Robo1. The position of the glycosylation site on Robo1 is located 
such that glycosylation could affect both domain-domain orientation and ligand binding. Here, we study a glycosylated form of the Robo1-Ig1-2 construct.

For the HS ligand, previous work has used natural isolates often from depolymerized heparin; in some cases, levels of sulfation have been chemically or enzymatically modified. ${ }^{11,12}$ Natural isolates are frequently inhomogeneous, making structural characterization difficult. Here, we capitalize on a synthetic strategy directed at the preparation of HS with specific sulfation patterns (Figure. 1B). ${ }^{11}$ Previous work has suggested that both 6- and $\mathrm{N}$-sulfation are important. ${ }^{7}$ We initially selected a tetramer containing both 6- and $\mathrm{N}$-sulfation, but also 2-O-sulfation on one of the IdoA residues.

Our approach to characterization using NMR has some unique aspects. Glycoproteins, like Robo1, often resist expression in the bacterial hosts used to produce uniformly isotopically labeled material for traditional NMR studies. Here, we use a sparse labeling strategy that can utilize expression in mammalian cells. ${ }^{13,14}$ Proteins can be labeled with a single isotopically labeled amino acid type or with small subsets of isotopically labeled amino acids. We select labeling with ${ }^{15} \mathrm{~N}$-lysine, because of the frequent involvement of this positively charged amino acid in interaction with negative sulfates and carboxylates on HS. We also label separately with ${ }^{15} \mathrm{~N}$-phenylalanine as it is sometimes found in glycan binding sites. Resonances from the labeled sites cannot be assigned by traditional sequential assignment methods. Therefore, an approach that combines chemical shift prediction with data from ${ }^{15} \mathrm{~N}$-edited nuclear Overhauser effects (NOEs) and residual dipolar couplings (RDCs) is used.

Fortunately, NMR resonances from a single lysine site shift upon adding the HS ligands, helping to locate the binding site. However, this site is unlikely to provide sufficient data to precisely dock the ligands in the binding site. Hence, we rely on transferred NOEs (trNOEs) and STD data to provide information on bound ligand geometry and ligand epitopes that contact the protein. These are well established techniques used extensively in the study of ligand-protein complexes. ${ }^{15}$ To provide more precise positioning in the binding site, we use long-range structural constraints from a paramagnetic tag added to a remote site in the protein. The particular tag is based on a lanthanide binding peptide designed by the Imperiali group ${ }^{16}$ and used in some of our previous work. ${ }^{17,18}$ Use of both peptide tags and chelate based tags is being used increasingly in the study of ligand-protein complexes. ${ }^{19,20} \mathrm{In}$ our case, perturbations of the sparsely labeled protein sites prove useful in positioning the tag and defining its susceptibility tensor. Once done, shifts of ligand resonances constrain its location in the protein's binding site. All of the NMR data have been combined in a constrained docking approach using HAD-DOCK. ${ }^{21}$ The docked structures provide a useful model for how HS modulates Slit2-Robo1 signaling.

\section{Results and Discussion}

\section{Chemical Shift Perturbation of Sparsely Labeled Robo1-lg1-2}

There are 12 lysines and five phenylalanines in the Robol-Ig1-2 construct. The $2 \mathrm{D}^{15} \mathrm{~N}-{ }^{1} \mathrm{H}$ heteronuclear single quantum coherence (HSQC) spectra of lysine and phenylalanine labeled Robo1-Ig1-2 are shown in Figure 2A and B, respectively. 
Crosspeaks for all phenylalanine residues are observed, but two crosspeaks are missing from the lysine HSQC. It is common to find missing crosspeaks due to high rates of amide proton exchange in solvent exposed regions or in regions that are dynamic. Comparing the lysine HSQC with that of a double mutant, R136A/K137A, that lacks binding activity, ${ }^{22}$ one finds a superimposable spectrum with no additional crosspeaks missing. This shows that one missing crosspeak belongs to K137. The other missing crosspeak belongs to K266. Mass spectral analysis shows K266 to be missing from our protein, possibly due to proteolysis during expression and isolation.

Chemical shift perturbation is a qualitative method for studying protein-ligand binding, allowing dissociation constants, $K_{\mathrm{d}}$, and binding site location to be determined. ${ }^{15} \mathrm{~N}-{ }^{1} \mathrm{H}$ HSQC spectra for lysine labeled Robo1-Ig1-2 in the presence of different concentrations of HS tetramers are overlaid in Figure 2A. One lysine residue shows a significant chemical shift perturbation. No phenylalanine residues show any significant perturbation during titration, implying that they are unlikely to be involved in the binding process. A binding affinity of $255 \pm 30 \mu \mathrm{M}$ has been extracted (Supporting Information Figure S1).

\section{Assignment of Sparsely Labeled Robo1-Ig1-2}

The new assignment strategy we introduce combines a number of NMR measurements that can be made on sparsely ${ }^{15} \mathrm{~N}$ labeled proteins (RDCs, NOEs, and chemical shifts) and compared with predictions based on the known domain structures of Robo1-Ig1-2. Since the individual measurement types seldom give unambiguous assignments, one would ideally examine predictions for all measurement types using an entire set of permuted assignments to make a decision. Even for the 10 unassigned lysine crosspeaks, this is an arduous task. Instead, we have sequentially applied each measurement type retaining only assignments that agree within generous error limits at each step. First, amide chemical shifts were predicted by the chemical shift prediction program, PPM_one, ${ }^{23}$ and compared to crosspeak positions; only sites giving a prediction within 2 times the estimated precision were retained. Next, NOEs for protons within $4 \AA$ of each labeled amide proton were predicted using distances extracted from structure $2 \mathrm{~V} 9 \mathrm{R}$ using Chimera ${ }^{24}$ and associated with shift positions predicted with PPM_one. These were compared to the experimental NOE measurements from the ${ }^{15} \mathrm{~N}$-edited NOESY experiment, again considering a shift difference of 2 times the precision $(0.34 \mathrm{ppm})$ to be in agreement and requiring observation of $2 / 3$ of the expected peaks. Lastly, RDCs were measured for each labeled site and compared with predictions using REDCAT. ${ }^{25}$ Our RDC predictions assume that a single rigid structural model can adequately represent the properties of Robo1-Ig $1-2$ in solution. This is potentially problematic since different interdomain orientations exist in various crystal forms. ${ }^{9,10}$ To find a reasonable model a long molecular dynamics (MD) run $(\sim 1 \mu \mathrm{s})$ was used to generate likely conformers and allow us to select a highly populated one. Solutions with RDC Q factors ${ }^{26}$ less than 0.3 were regarded as acceptable.

The final assignments are listed in Table 1, and the details of the sequential elimination process are summarized in Supporting Information Table S1. Two additional pieces of information were used to confirm assignments and resolve ambiguities: the consistency with distance information from a Robo1-Ig1-2 loop construct containing $\mathrm{Gd}^{3+}$ was examined 
(see section on paramagnetic perturbations); and a construct containing only the first domain was expressed and analyzed to make a correct association of crosspeaks with each of the two domains.

Most important among these assignments is that of crosspeak 6 (122.7 ppm, $8.6 \mathrm{ppm}$ ); this shifts with the addition of the ligand and is assigned to K81. Significantly, an HS tetramer lacking a 2-O-sulfate shows a much smaller shift of K81 (Supporting Information Figure $\mathrm{S} 2$ ), suggesting that $\mathrm{K} 81$ is involved in a direct interaction with this sulfate group. This, along with abolition of binding in the R136A/K137A mutant, points to a potential binding site on the protein.

\section{Saturation Transfer Difference NMR}

Saturation transfer difference (STD) experiments complement the chemical shift perturbation experiments nicely in that they identify potential interaction epitopes on the ligands. The saturation of magnetization of protons on the protein is transferred to protons on the ligand in a distance dependent way, and resonances from ligand protons with a close approach to a protein proton will decrease in intensity. A difference spectrum collected with and without saturation leaves primarily ligand resonances experiencing this transfer. There are, however, some complexities of importance to glycoproteins. Usually a spin- spin relaxation filter is used to remove the broad protein resonances. For a glycoprotein, resonances from the attached glycan are not broad and persist in the difference spectrum at positions that often overlap with those of our ligand. Therefore, a double difference spectrum was produced using an STD spectrum acquired on the protein in the absence of ligand. The largest STD signals arise from $\mathrm{H} 2 \mathrm{C}$ (or $\mathrm{H} 2 \mathrm{~A}$ which is overlapped), $\mathrm{H} 2 \mathrm{~B}$, and $\mathrm{H} 4 \mathrm{D}$ (see Figure 1B for residue designation; proton numbering begins at the anomeric carbon for each residue). H2B is on the 2-sulfated IdoA residue, supporting a direct interaction of this residue with the protein surface near K81. Most other protons show significant signals, suggesting a significant spin diffusion among ligand protons. Thus, we will confine our use to the three protons mentioned above. Both build-up curves and a histogram summary of all STD data are provided in Supporting Information Figures S3 and S4.

\section{Transferred Nuclear Overhauser Effects}

Heparan sulfates are a group of glycans with a significant degree of internal mobility, in terms of variations in both glycosidc bond torsion angles and iduronic acid ring forms. A subset of these conformers is likely to be selected upon binding to Robo1- Ig1-2. Like NOEs, trNOEs provide insight into conformations sampled through their $1 / I^{6}$ dependence on interproton distances between pairs of ligand protons. In general, conformers from both the free ligand in solution and the bound ligand are sampled, and an average trNOE is measured. However, transfer of magnetization in large molecular assemblies is far more efficient than in small molecules. This allows contributions to trNOEs from bound ligands to dominate over contributions from ligands in solution. The contributions from free ligands can be further reduced by taking advantage of the shift from positive to negative NOEs as temperatures decrease and rotational correlation times increase. At $27{ }^{\circ} \mathrm{C}$ and $900 \mathrm{MHz}$, where we collected trNOE data, correlation times decrease sufficiently for the free HS 
tetramer to bring its contributions near zero. Hence, trNOEs taken at $27{ }^{\circ} \mathrm{C}$ can be converted directly to bound ligand distances using a reference NOE having a known fixed distance, in our case, that for the GlcNAc H2-H4 pair (2.5 $)$. Distances converted in this way are still weighted averages of bound state conformations. However, the number of conformers sampled in the bound state tends to be small, and derived distances should be close to those in the minimum energy bound conformer. The derived distances between pairs of nuclei on opposite sides of the glycosidic bonds are listed in Table 2.

For comparison, distances derived from NOEs for the same pairs in the absence of protein, and at $15{ }^{\circ} \mathrm{C}$ where free ligand NOEs are again measurable, are also listed. There are some differences between the bound and free states. For example, the distance between GlcNAc A $\mathrm{H} 4$ and IdoA B H1 in the bound state is $1.90 \pm 0.04$, while that found in the free state is 2.38 $\pm 0.05 \AA$. This deviation involves the terminal residues of the tetrasaccharide which may have more motional freedom in solution; no significant differences were observed for the central portion of the tetrasaccharide, suggesting that something close to the minimum energy conformer found in solution is selected for the bound state.

\section{Pseudo Contact Shifts (PCSs) of Robo1-lg1-2 and the HS Complex}

Paramagnetic effects caused by lanthanide ions offer unique opportunities to more quantitatively position ligands in protein-ligand complexes. ${ }^{27}$ PCSs are caused by an average magnetic field from an induced dipole moment centered on the unpaired electron distribution of the lanthanide. It depends not only on the distance between a nucleus and the metal ion (decreases with $1 / r^{3}$ ) but also on the orientation and magnitude of the anisotropic part of the ion's susceptibility tensor. To provide a site capable of binding a paramagnetic ion in Robo1-Ig1-2, a short polypeptide (SYIDTNNDGAYEGDELSG) has been engineered into the Robo1- $\operatorname{Ig} 1-2$ construct between strands C and D of Ig1. Luminescence data based on a tryptophan to $\mathrm{Tb}^{3+}$ energy transfer show the site to have an ion binding affinity of 62 $\mathrm{nM}$ (Supporting Information Figure S5). PCS data for the Lys and Phe labeled Robo1-Ig1-2 protein are shown in Figure 3. The unique diagonal shifts in peak positions from the superimposed spectra with paramagnetic $\left(\mathrm{Tm}^{3+}\right)$ and diamagnetic $\left(\mathrm{Lu}^{3+}\right)$ ions are used to pair the resonances in each spectrum. Similar measurements can be made on well resolved resonances from ligands in 1D proton experiments. In these cases, an average of the resonance position for the uncomplexed and complexed ligand is measured, and shifts are scaled by the percentage bound (Table 3).

\section{Location and Tensor Alignment for the Ln-Binding Loop}

Before the observed PCSs for the ligand can be converted to useful constraints on ligand position in the binding site, the position of the lanthanide ion and its effective susceptibility tensor must be determined. This involves specifying five independent elements of the tensor plus positional coordinates. The latter were obtained by averaging $\mathrm{Ln}^{3+}$ positions found in an extensive MD simulation. For the tensor element determination it would be tempting to use PCSs for all 15 labeled protein residues. However, we would need to assume a rigid Robo1-Ig1-2 structure, and previous literature indicates significant flexibility between Robo1 Ig1 and Ig2 domains. ${ }^{9,10}$ Using data for just domain Ig1, there are three lysines and three phenylalanines, making the number of data points for tensor determination marginal. 
Hence, we used the fact that the same anisotropic part of the susceptibility tensor is responsible for both the PCSs and field induced RDCs. The Q factor for back-calculated RDC plus PCS data from the Ig1 domain was 0.22 (Pearson correlation coefficient or $R$ factor of 0.96), indicating a good fit. We can also use the derived tensor, along with the complete set of RDC and PCS data, to test the suitability of the rigid model used in our resonance assignment protocol. The back-calculated data for the Ig2 domain (minus one point from a loop region) fit measurements with a reasonable $R$ factor of 0.70 . While this is not as good as expected for a completely rigid model, it does suggest that the model selected is well populated. Therefore, we will continue to use this model in ligand docking studies to be described below. The tensor and all data used for tensor determination are listed in Supporting Information Table S2.

\section{Computational Docking}

High ambiguity driven bio-molecular docking (HADDOCK) ${ }^{21}$ was used to combine all of the structural constraints and determine the structure of a Robo1-Ig1-2-HS complex. More qualitative information, such as that coming from mutagenesis and STD experiments, is represented as ambiguous restraints and the more quantitative ones, such as the specific interaction between $\mathrm{K} 81$ and the 2-O-sulfate of the HS tetramer and those coming from trNOE and PCSs, are treated explicitly in error functions that compare experimental data and predictions calculated from various trial structures. In the end, the top 5 HADDOCK structures with the lowest energies among the 20 structures with the highest scores and no distance restraint violations greater than $0.5 \AA$ are shown in Figure 4A.

The structures show a well-clustered binding location as well as well-defined ligand conformation. All of the IdoA residues prefer a ${ }^{1} \mathrm{C}_{4}$ chair conformation. Starting structures with ligand residues in both the chair ${ }^{4} \mathrm{C}_{1}$ and the skew-boat ${ }^{2} \mathrm{~S}_{0}$ conformation were tested. Neither gave clusters with competitive scores or energies. The ${ }^{1} \mathrm{C}_{4}$ chair conformation is also known to be more energetically favorable in solution especially when it is at the nonreducing terminus. ${ }^{28}$

The structure with the lowest energy is used as an illustration in Figure 4B: residues within 1 $\AA$ of van der Waals contact of the ligand include K81, V133, H134, G135, R136, K137, I167, and R169. Five of these are positively charged residues, a number close to the number of negatively charged residues in the HS tetramer (7). The pairwise electrostatic interactions and the distances between charged entities are summarized in Table 4 .

There are also strong van der Waals interactions that may explain some of the STD signals. The epsilon methylene protons of $\mathrm{K} 81$ are close enough to $\mathrm{H} 2$ of IdoA B for at least transient interactions $(2.7 \AA)$. The gamma methyl protons of I167 are in van der Waals contact with $\mathrm{H} 4$ of IdoA D, and the methyl protons of V133 are in van der Waals contact with the reducing terminus extension on GlcNAc A.

\section{Analysis of Binding Energy Contributions}

It is tempting to focus on the electrostatic interactions as the primary origin of binding affinities and ascribe particular importance to the K81-IdoA-B 2-O-sulfate interaction because of the pronounced change in chemical shift of the K81 crosspeak. However, one 
must remember that electrostatic interactions with other polar groups, as well as van der Waals terms contribute, and that affinities are the result of differences in energies of interaction in the complex, and interactions of separated protein and ligand with solvent. Molecular mechanics-generalized Born surface area (MM-GBSA) calculations provide one way of looking at affinities with a broader perspective. ${ }^{29,30}$ We preformed these calculations on the top 5 docked structures, excluding conformational entropy terms. The use of just the docked structures implicitly assumes that the conformations of separated protein and ligand remain as in the complex. This certainly results in an overestimation of binding energies, but since we are primarily concerned with relative contributions of different residues, this is of minor importance. A per-residue decomposition was performed, ${ }^{31}$ treating the 2 -O-sulfate and 6-O-sulfates as separate residues so their contributions could be explicitly examined. For the IdoA-B 2-O-sulfate, the electrostatic contribution averaged over the top five structures is large $\left(-49.4 \mathrm{kcal} \mathrm{mol}^{-1}\right)$. However, this is more than offset by the high desolvation penalty $\left(52.3 \mathrm{kcal} \mathrm{mol}^{-1}\right)$. Even when nonpolar solvation and van der Waals terms are included, there is a net unfavorable energy of association of $1.33 \mathrm{kcal}$. Similar observations pertain to the 6-O-sulfates, but the unfavorable energies are less $(0.8$ and $1.1 \mathrm{kcal})$. The sugar rings themselves, which contain a negatively charged carboxylate in the case of IdoA residues and $\mathrm{N}$-sulfates in the case of the two GlcNAc residues, all have negative energy estimates, resulting in a total HS tetramer energy of $-0.2 \mathrm{kcal}$. The protein residues that interact with the HS tetramer make larger and more favorable contributions to the energy differences between complexed and free states, primarily because the protein residues are less exposed and less solvated in the free state. Adding protein residue contributions to HS residue contributions, the 2-O-sufate on IdoA B, actually makes a smaller contribution to binding than the 6-O-sulfate on GlcNAc C. This does not mean that the interaction of K81 with the 2-O-sulfate is insignificant. If it did not occur, much of the 2-O-sulfate desolvation penalty would still exist, affecting either binding geometries or affinities. However, the analysis does emphasize the importance of considering all interactions, both in complexed and free states, before ascribing importance to any single aspect of a structural model.

\section{Comparison to Other Structures}

To date, there have been several structural characterizations of Robo1-ligand interactions using different methodologies. ${ }^{7,9,10,22}$ It is of particular interest to compare our results to the crystal structure of drosophila Robo1-Ig1-2 in which a heparin tetramer has been modeled. The protein sequence is $53 \%$ identical, and the HS tetramers are identical except that the heparin fragment has both IdoA residues 2-O-sulfated. In the crystal structure, the heparin fragment is sandwiched between two Robo1-Ig1-2 monomers. The residue corresponding to K81 in our structure is involved with binding in both monomers. However, in our experiment, an average correlation time of $13 \mathrm{~ns}$ measured from cross-correlation experiments of Robo1 alone and the Robo1-Ig1-2-HS complex reveals that the protein remains monomeric before and after interacting with the HS tetramer in solution (Supporting Information Figure S6). Moreover, there is a single glycosylation site near the C-terminus of domain Ig1 that may well influence dimerization and interdomain geometry. Most crystal structures have employed material lacking this glycosylation. 
Crystal structures also show different positions of domain Ig2 relative to Ig $1 .{ }^{9,10}$ From our MD simulation, the protein tends to adopt a more bent conformation after the first $400 \mathrm{~ns}$ of stabilization. This conformation was used in our initial assignment strategy and its use supported by our PCS/RDC back-calculations. The curved structure is able to make more efficient contact between the interacting amino acid residues and each glycan ring than the straight form, particularly I167 and R169.

\section{Modeling a Robo1-HS-Slit2 Complex}

One of the driving principles behind determination of a structure for a complex of Robo1 with a well-defined, but relatively short HS oligomer, is the potential for using this structure to understand interactions of Robo1 with longer HS oligomers and its signaling partners. Robo1 signaling is initiated by interactions with Slit2, and this interaction is known to be facilitated by interactions with HS. In Figure 4C, we have superimposed Robo1-Ig1 in our model with Robo1-Ig1, an existing crystal structure of the Robo1-Ig1-Slit2-D2 complex. ${ }^{10}$ We have also extended our tetrasaccharide by a GlcA-GlcNS6S unit in each direction. The resulting HS octamer sits well in the groove between the two proteins, and some of the positively charged residues highlighted in red on both Robo1 and Slit2 show potential interaction sites by which a longer HS segment could stabilize the trimeric complex.

\section{Conclusions}

A detailed model for the interaction of a synthetic HS tetramer with a two domain fragment of Robo1 has been determined. The model leads to a plausible explanation for how HS facilitates the interaction between Robo1 and its signaling partner, Slit2, providing a guide for further studies using longer HS oligomers and complexes involving both Robo1 and Slit2. The methods used in the current study also set a precedent for studies of other complexes of glycosylated proteins. The methods used exploit a number of NMR experiments that can be applied to glycosylated proteins sparsely labeled with NMR active isotopes and should be applicable to the large number of other systems found on the surfaces of mammalian cells.

\section{Methods}

Materials

The HS tetramers were synthesized using methodology previously described. ${ }^{11}{ }^{15} \mathrm{~N}$ Phe, ${ }^{15} \mathrm{~N}$ - Lys, and deuterium oxide were purchased from Cambridge Isotope Laboratories. All other chemicals were purchased from Sigma-Aldrich unless otherwise stated.

\section{Protein Expression and Purification}

The Robo1-Ig1-2 construct containing a lanthanide binding loop was chosen based on the similarity in separation of strand ends and loop ends in a previous insert in an a-helical protein. ${ }^{18}$ The final sequence with the inserted tag in bold is GSRLRQEDFPPRIVEHPSDLIVSKGEPATLNCKAEGRPTPTIEWYKGSYIDTNNDGAY EGDELSGGERVETDKDDPRSHRMLLPSGSLFFLRIVHGRKSRPDEGVYVCVARNYL GEAVSHNASLEVAILRDDFRQNPSDVMVAVGEPAVMECQPPRGHPEPTISWKKDGSP LDDKDERITIRGGKLMITYTRKSDAGKYVCVGTNMVGERESEVAELTVLERPSFVK. 
The detailed expression and purification procedure was described previously ${ }^{13}$ except the cell expression medium was exchanged to a custom Freestyle dropout medium supplemented with $150 \mathrm{mg} \mathrm{L}^{-1}$ isotopically labeled Phe or Lys on the second day of transfection. The average protein yields were $10 \mathrm{mg} \mathrm{L}^{-1}$. Examination of trypsinized fragments containing lysine and phenylalanine indicate that ${ }^{15} \mathrm{~N}$ labeling is $75 \%$ efficient at these amino acids. $\mathrm{N}$-linked oligosaccharides were released by treatment with PNGase F and analyzed by ESI-MS/MS (LTQ-Orbitrap, Thermo Scientific). The glycans are very heterogeneous with most major peaks belonging to biantennary structures having core fucosylation (Supporting Information Figure S7).

\section{NMR Spectroscopy}

All the NMR spectroscopy was performed on Varian/Agilent instruments with DD2 (21.1 and $18.8 \mathrm{~T}$ ) consoles and $5 \mathrm{~mm}$ cryogenically cooled triple resonance probes. NMR protein samples were $150 \mu \mathrm{M}$ in $10 \%$ (v/v) $\mathrm{D}_{2} \mathrm{O}$ buffer containing $25 \mathrm{mM}$ Tris and $100 \mathrm{mM} \mathrm{KCl}$ at pH 7.0 for ${ }^{15} \mathrm{~N}$ HSQC titrations, 3D ${ }^{15} \mathrm{~N}$-filtered NOE experiments, and RDC experiments. Robo1 loop samples contained lanthanides at lanthanide to protein ratios slightly less than 1:1. Pseudo contact shifts (PCSs) of proteins and ligands were determined from standard HSQC spectra with 1:2 protein/ligand ratios. Samples for the STD and trNOE experiments were $15 \mu \mathrm{M}$ in protein and $900 \mu \mathrm{M}$ in ligand, all in $100 \%$ (v/v) $\mathrm{D}_{2} \mathrm{O}$ buffer containing 20 $\mathrm{mM}$ phosphate and $100 \mathrm{mM} \mathrm{KCl}$ at $\mathrm{pH}$ 7.0. All samples contained dimethyl-2silapentane-5-sulfonate (DSS) as an internal reference.

NMR experiments were standard Biopack experiments conducted at $27{ }^{\circ} \mathrm{C}$, except for the NOE experiment on the free ligand which was at $15^{\circ} \mathrm{C}$. A mixing time of $150 \mathrm{~ms}$ was used in trNOE and HSQC-NOESY experiments; $500 \mathrm{~ms}$ was used for the free ligand NOE experiment. RDCs were measured on a protein sample containing $12.5 \mathrm{mg} \mathrm{mL}^{-1} \mathrm{Pf} 1$ phage (ASLA biotech) using a pulse sequence in which cross-peaks in HSQC spectra are modulated by $\mathrm{J}+\mathrm{D}$ coupling in the ${ }^{15} \mathrm{~N}$ dimension. ${ }^{32}$ For STD, both the protein only and complex samples were irradiated at $-1.5 \mathrm{ppm}$, and saturation times were increased from 1 to $4 \mathrm{~s}$ in steps of $1 \mathrm{~s}$. The assignment of ligand proton resonances was accomplished by acquiring ${ }^{1} \mathrm{H}$ proton, ${ }^{1} \mathrm{H}-{ }^{1} \mathrm{H}$ TOCSY, ${ }^{1} \mathrm{H}-{ }^{1} \mathrm{H}$ NOESY, ${ }^{13} \mathrm{C}-{ }^{1} \mathrm{H}$ HSQC , and ${ }^{13} \mathrm{C}-{ }^{1} \mathrm{H}$ HMQC spectra. Complete assignments are included in Supporting Information Figure S8. All the NMR data were processed with NMRPipe ${ }^{33}$ and SPARKY.${ }^{34}$ Chemical shift perturbations were fit to determine binding constants as described in the literature. ${ }^{35}$

\section{Susceptibility Tensor Determination}

To extract susceptibility tensors needed in calculating PCS derived constraints on the ligand, experimental RDCs and PCSs for ${ }^{15} \mathrm{~N}$ labeled protein sites were used. RDCs $\left(\mathrm{D}_{\mathrm{NH}, \exp (900)}\right)$ were extracted for the sites in domain $\operatorname{Ig} 1$ using the formula in eq 1 and data collected at field strengths corresponding to proton observation at 600 and $900 \mathrm{MHz}$. $J$ and $D$ are scalar and dipolar coupling contributions, respectively. 


$$
D_{\mathrm{NH}, \exp (900)}=\frac{900^{2}}{900^{2}-600^{2}}\left[(J+D)_{\mathrm{HN}(900)}-(J+D)_{\mathrm{HN}(600)}\right]
$$

The PCSs and RDCs were then combined in the program REDCAT to determine the susceptibility tensor. This requires appropriate scaling using different RDCmax and PCSmax constants $\left(24350 \mathrm{~Hz}\right.$ for ${ }^{15} \mathrm{~N}-{ }^{1} \mathrm{H}$ RDCs and $18.54 \times 10^{6} \mathrm{ppm}$ for PCSs at $\left.900 \mathrm{MHz}\right)$.

\section{Simulation of the Robo1-lg1-2-Loop Construct}

To provide an appropriate PDB file for loop containing Robo1-Ig1-2, a molecular dynamics (MD) trajectory was produced. It was carried out using the AMBER 14 package $^{36}$ and the ff14SB force field ${ }^{37}$ with the SANDER module. The GLYCAM_06j-1 force field ${ }^{38}$ was adopted for carbohydrate simulation. PDB $2 \mathrm{v} 9 \mathrm{r}$ was used to obtain the initial atomic coordinates, and the lanthanide-binding loop was modeled in using tools in CHIMERA. ${ }^{24} \mathrm{~A}$ cubic box of TIP3 $\mathrm{P}^{39}$ water was used to solvate the protein. The system was first energy minimized by 2000 steps of minimization, then heated to $300 \mathrm{~K}$ at 2 fs stepwise for $400 \mathrm{ps}$. The MD simulation lasted for $1 \mu \mathrm{s}$. Frames from 401 to $1000 \mathrm{~ns}$ were used to find the average ion and isotopically labeled site positions required by REDCAT.

\section{Robo1-HS Complex Assembly by HADDOCK}

Models of Robo1-HS complex were generated using the docking program HADDOCK. ${ }^{21}$ The average Robo1-Ig1-2-loop structure generated from MD simulation was used as the input protein structure. Ligand structures were produced using the GLYCAM web server. ${ }^{40}$ Restraints involving residues of the protein or parts of the ligand identified as being involved in an interaction by chemical shift perturbation, mutagenesis, or STD intensity were entered as ambiguous interaction restraints. Interproton distances derived from trNOE data were converted to upper and lower bounds for distance constraints by adding or subtracting $0.3 \AA$. A distance constraint involving the 2-sulfate of the ligand and K81 of the protein was implemented in a similar manner to NOEs with a lower bound at van der Waals contact and an upper bound at $4 \AA$ A PCSs data for both the protein and ligand were implemented using the XPCS restraints. All the restraints are listed in the Supporting Information Table S4. The ligand was set to be fully flexible, and the loops of the protein containing the residues having the most perturbed chemical shift on ligand addition or identified as interacting residues in mutational studies were specified as semiflexible. The loop connecting the Ig1 and Ig2 is among the semi-flexible regions. The docking began with rigid-body energy minimization followed by semiflexible refinement using simulated annealing and ended with water refinement. A total of 200 refined models ranked by HADDOCK score were obtained, and the 20 top scoring models were ordered by total energy.

\section{Energy Calculations}

Similar MD trajectories were initiated as described for the Robo1-Ig1-2-loop construct, but now with the HS tetrasaccharide docked into the Robo1-Ig1-2-loop construct as in the top five results from HADDOCK. Production runs of $50 \mathrm{~ns}$ were initiated after $50 \mathrm{ps}$ of minimization, heating, and density equilibration and $2200 \mathrm{ps}$ of constant pressure 
equilibration at $300 \mathrm{~K}$. In order to calculate the free energy of the bound state and the solvation energy of the protein and ligand in solvent, the molecular mechanics generalized Born surface area (MM-GBSA) method ${ }^{29,30}$ as implemented in AMBER-14 ${ }^{36}$ was used. This was followed by per-residue decomposition as described elsewhere. ${ }^{41}$

\section{Supplementary Material}

Refer to Web version on PubMed Central for supplementary material.

\section{Acknowledgments}

We thank R. Sonon for his kind assistance in N-glycosylation profiling. We also thank A. Singh and D. Thieker for their advice in MD simulation and post energy analysis. This work was supported by a grant from the National Institutes of Health, P41GM103390. The content is solely the responsibility of the authors and does not necessarily represent the official views of the National Institutes of Health.

\section{References}

1. Kastenhuber E, Kern U, Bonkowsky JL, Chien CB, Driever W, Schweitzer J. Netrin-DCC, RoboSlit, and Heparan Sulfate Proteoglycans Coordinate Lateral Positioning of Longitudinal Dopaminergic Diencephalospinal Axons. J Neurosci. 2009; 29:8914-8926. [PubMed: 19605629]

2. Andrews W, Liapi A, Plachez C, Camurri L, Zhang JY, Mori S, Murakami F, Parnavelas JG, Sundaresan V, Richards LJ. Robo1 regulates the development of major axon tracts and interneuron migration in the forebrain. Development. 2006; 133:2243-2252. [PubMed: 16690755]

3. Domyan ET, Branchfield K, Gibson DA, Naiche LA, Lewandoski M, Tessier-Lavigne M, Ma L, Sun X. Roundabout Receptors Are Critical for Foregut Separation from the Body Wall. Dev Cell. 2013; 24:52-63. [PubMed: 23328398]

4. Dickinson RE, Duncan WC. The SLIT-ROBO pathway: a regulator of cell function with implications for the reproductive system. Reproduction. 2010; 139:697-704. [PubMed: 20100881]

5. (5) Gara RK, Kumari S, Ganju A, Yallapu MM, Jaggi M, Chauhan SC. Slit/Robo pathway: a promising therapeutic target for cancer. Drug Discovery Today. 2015; 20:156-164. [PubMed: 25245168]

6. Hussain SA, Piper M, Fukuhara N, Strochlic L, Cho G, Howitt JA, Ahmed Y, Powell AK, Turnbull JE, Holt CE, Hohenester E. A molecular mechanism for the heparan sulfate dependence of SlitRobo signaling. J Biol Chem. 2006; 281:39693-39698. [PubMed: 17062560]

7. Zhang FM, Moniz HA, Walcott B, Moremen KW, Linhardt RJ, Wang LC. Characterization of the interaction between Robo1 and heparin and other glycosaminoglycans. Biochimie. 2013; 95:23452353. [PubMed: 23994753]

8. Dickson BJ, Gilestro GF. Regulation of commissural axon pathfinding by slit and its Robo receptors. Annu Rev Cell Dev Biol. 2006; 22:651-675. [PubMed: 17029581]

9. Fukuhara N, Howitt JA, Hussain SA, Hohenester E. Structural and functional analysis of slit and heparin binding to immunoglobulin-like domains 1 and 2 of Drosophila Robo. J Biol Chem. 2008; 283:16226-16234. [PubMed: 18359766]

10. Morlot C, Thielens NM, Ravelli RBG, Hemrika W, Romijn RA, Gros P, Cusack S, McCarthy AA. Structural insights into the Slit-Robo complex. Proc Natl Acad Sci U S A. 2007; 104:1492314928. [PubMed: 17848514]

11. Zong CL, Venot A, Dhamale O, Boons GJ. Fluorous Supported Modular Synthesis of Heparan Sulfate Oligosaccharides. Org Lett. 2013; 15:342-345. [PubMed: 23293947]

12. Chappell EP, Liu J. Use of biosynthetic enzymes in heparin and heparan sulfate synthesis. Bioorg Med Chem. 2013; 21:4786-4792. [PubMed: 23313092]

13. Barb AW, Meng L, Gao ZW, Johnson RW, Moremen KW, Prestegard JH. NMR Characterization of Immunoglobulin G Fc Glycan Motion on Enzymatic Sialylation. Biochemistry. 2012; 51:46184626. [PubMed: 22574931] 
14. Prestegard JH, Agard DA, Moremen KW, Lavery LA, Morris LC, Pederson K. Sparse labeling of proteins: Structural characterization from long range constraints. J Magn Reson. 2014; 241:32-40. [PubMed: 24656078]

15. Roldos V, Canada FJ, Jimenez-Barbero J. Carbohydrate-Protein Interactions: A 3D View by NMR. Chem-BioChem. 2011; 12:990-1005.

16. Barthelmes K, Reynolds AM, Peisach E, Jonker HRA, DeNunzio NJ, Allen KN, Imperiali B, Schwalbe H. Engineering Encodable Lanthanide-Binding Tags into Loop Regions of Proteins. J Am Chem Soc. 2011; 133:808-819. [PubMed: 21182275]

17. Zhuang TD, Lee HS, Imperiali B, Prestegard JH. Structure determination of a Galectin-3carbohydrate complex using paramagnetism-based NMR constraints. Protein Sci. 2008; 17:12201231. [PubMed: 18413860]

18. Barb AW, Ho TG, Flanagan-Steet H, Prestegard JH. Lanthanide binding and IgG affinity construct: Potential applications in solution NMR, MRI, and luminescence microscopy. Protein Sci. 2012; 21:1456-1466. [PubMed: 22851279]

19. Liu WM, Overhand M, Ubbink M. The application of paramagnetic lanthanoid ions in NMR spectroscopy on proteins. Coord Chem Rev. 2014; 273:2-12.

20. Kato K, Yamaguchi T. Paramagnetic NMR probes for characterization of the dynamic conformations and interactions of oligosaccharides. Glycoconjugate J. 2015; 32:505-513.

21. Dominguez C, Boelens R, Bonvin AMJJ. HADDOCK: A protein-protein docking approach based on biochemical or biophysical information. J Am Chem Soc. 2003; 125:1731-1737. [PubMed: 12580598]

22. Li ZX, Moniz H, Wang S, Ramiah A, Zhang FM, Moremen KW, Linhardt RJ, Sharp JS. High Structural Resolution Hydroxyl Radical Protein Footprinting Reveals an Extended Robo1-Heparin Binding Interface. J Biol Chem. 2015; 290:10729-10740. [PubMed: 25752613]

23. Li DW, Bruschweiler R. PPM_One: a static protein structure based chemical shift predictor. J Biomol NMR. 2015; 62:403-409. [PubMed: 26091586]

24. Pettersen EF, Goddard TD, Huang CC, Couch GS, Greenblatt DM, Meng EC, Ferrin TE. UCSF chimera - A visualization system for exploratory research and analysis. J Comput Chem. 2004; 25:1605-1612. [PubMed: 15264254]

25. Valafar H, Prestegard JH. REDCAT: a residual dipolar coupling analysis tool. J Magn Reson. 2004; 167:228-241. [PubMed: 15040978]

26. Lipsitz RS, Tjandra N. Residual dipolar couplings in NMR structure analysis. Annu Rev Biophys Biomol Struct. 2004; 33:387-413. [PubMed: 15139819]

27. Otting G. Protein NMR Using Paramagnetic Ions. Annu Rev Biophys. 2010; 39(39):387-405. [PubMed: 20462377]

28. Ferro DR, Provasoli A, Ragazzi M, Casu B, Torri G, Bossennec V, Perly B, Sinay P, Petitou M, Choay J. Conformer Populations of L-Iduronic Acid Residues in Glycosamino-glycan Sequences. Carbohydr Res. 1990; 195:157-167. [PubMed: 2331699]

29. Kollman PA, Massova I, Reyes C, Kuhn B, Huo SH, Chong L, Lee M, Lee T, Duan Y, Wang W, Donini O, Cieplak P, Srinivasan J, Case DA, Cheatham TE. Calculating structures and free energies of complex molecules: Combining molecular mechanics and continuum models. Acc Chem Res. 2000; 33:889-897. [PubMed: 11123888]

30. Gandhi NS, Mancera RL. Free energy calculations of glycosaminoglycan-protein interactions. Glycobiology. 2009; 19:1103-1115. [PubMed: 19643843]

31. Gohlke H, Case DA. Insights into protein-protein binding by binding free energy calculation and free energy decomposition using a generalized born model. Abstr, Pap, Am Chem Soc. 2003; 225:U791-U791.

32. Tjandra N, Grzesiek S, Bax A. Magnetic field dependence of nitrogen-proton J splittings in N-15enriched human ubiquitin resulting from relaxation interference and residual dipolar coupling. $\mathrm{J}$ Am Chem Soc. 1996; 118:6264-6272.

33. Delaglio F, Grzesiek S, Vuister GW, Zhu G, Pfeifer J, Bax A. Nmrpipe - a Multidimensional Spectral Processing System Based on Unix Pipes. J Biomol NMR. 1995; 6:277-293. [PubMed: 8520220]

34. Goddard, T.; Kneller, D. Sparky. Vol. 3. University of California; San Francisco: 2004. 
35. Williamson MP. Using chemical shift perturbation to characterise ligand binding (vol 73, pg 1, 2013). Prog Nucl Magn Reson Spectrosc. 2014; 80:64-64.

36. Case, D.; Babin, V.; Berryman, J.; Betz, R.; Cai, Q.; Cerutti, D.; Cheatham Iii, T.; Darden, T.; Duke, R.; Gohlke, H. Amber. Vol. 14. University of California; San Francisco, CA: 2014.

37. Case, D.; Cai, Q.; Cerutti, D.; Cheatham, T., III; Darden, T.; Duke, R.; Gohlke, H.; Goetz, A.; Gusarov, S. Amber. Vol. 14. University of California; San Francisco, CA: 2014. The FF14SB force field; p. 29-31.

38. Kirschner KN, Yongye AB, Tschampel SM, Gonzalez-Outeirino J, Daniels CR, Foley BL, Woods RJ. GLYCAM06: A generalizable Biomolecular force field. Carbohydrates J Comput Chem. 2008; 29:622-655. [PubMed: 17849372]

39. Sattelle BM, Almond A. Less is More When Simulating Unsulfated Glycosaminoglycan 3DStructure: Comparison of GLYCAM06/TIP3P, PM3-CARB1/TIP3P, and SCC-DFTB-D/ TIP3P Predictions With Experiment. J Comput Chem. 2010; 31:2932-2947. [PubMed: 20564659]

40. Woods, R. Complex Carbohydrate Research Center. University of Georgia; Athens, GA: 2005. glycam Web.

41. Gohlke H, Kiel C, Case DA. Insights into protein-protein binding by binding free energy calculation and free energy decomposition for the Ras-Raf and Ras-RaIGDS complexes. J Mol Biol. 2003; 330:891-913. [PubMed: 12850155] 


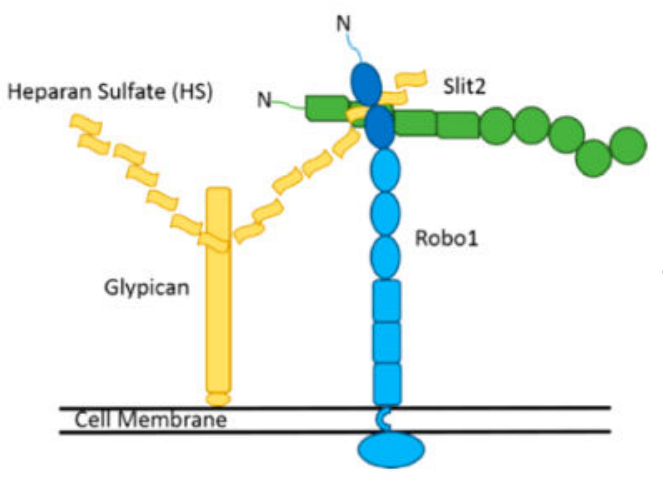

A

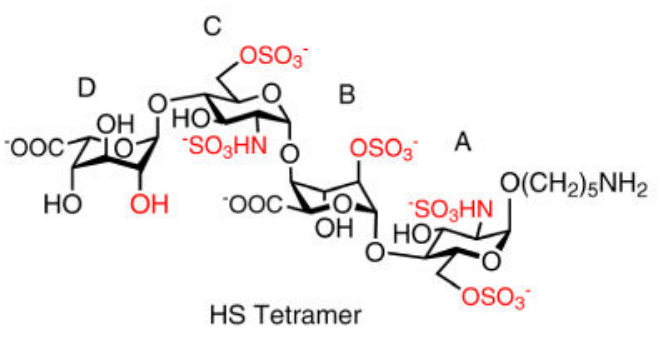

B

Figure 1.

(A) Cartoon representation of the Robo1-Slit2-HS interaction. Robo1 is shown in blue, with the Ig1-2 domains in dark blue. Slit2 is in green, and the heparan sulfate chain attached to glypican is in yellow. (B) Structure of the heparan sulfate tetramer used in this study with sulfate groups labeled in red. 


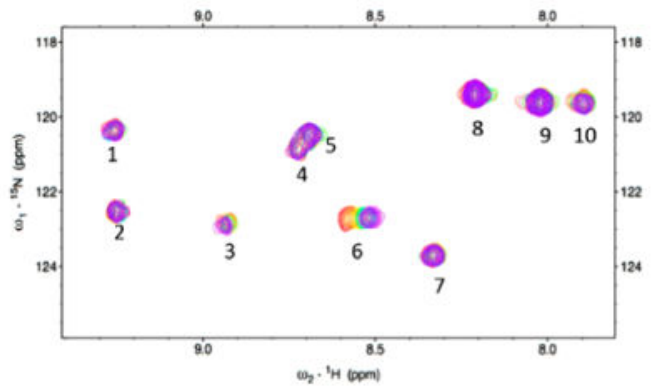

A

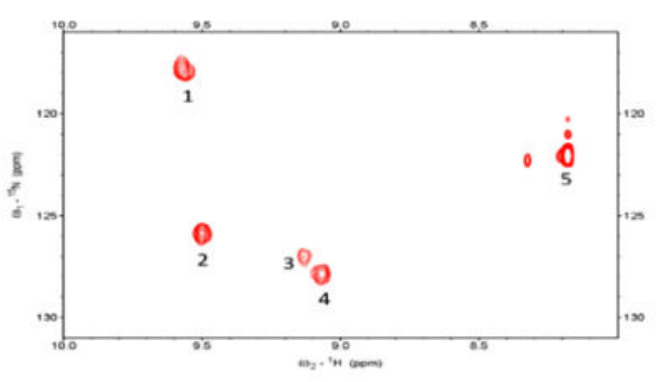

B

Figure 2.

2D ${ }^{15} \mathrm{~N}-{ }^{1} \mathrm{H}$ HSQC spectra of (A) ${ }^{15} \mathrm{~N}$ Lys labeled Robo1-Ig1-2 with HS tetramer titration and (B) ${ }^{15} \mathrm{~N}$-Phe labeled Robo1-Ig1-2. The protein is at $140 \mu \mathrm{M}$ in a pH 7 buffer at $27{ }^{\circ} \mathrm{C}$. Each crosspeak is labeled with an arbitrary number; assignments will follow. Overlaid spectra in A are color coded with increasing concentration of HS tetramer $(0 \mu \mathrm{M}$ of ligand in red and $560 \mu \mathrm{M}$ in purple with a stepwise increase of $70 \mu \mathrm{M}$ ). 


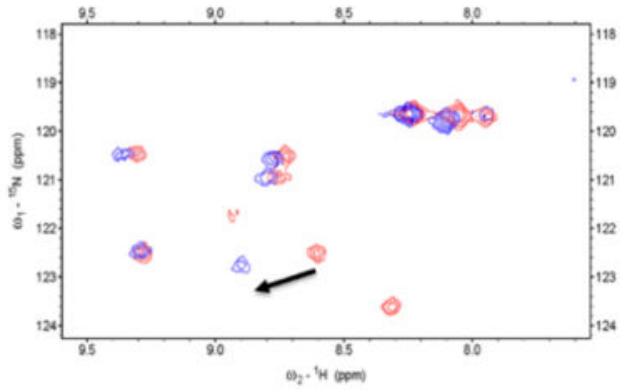

A

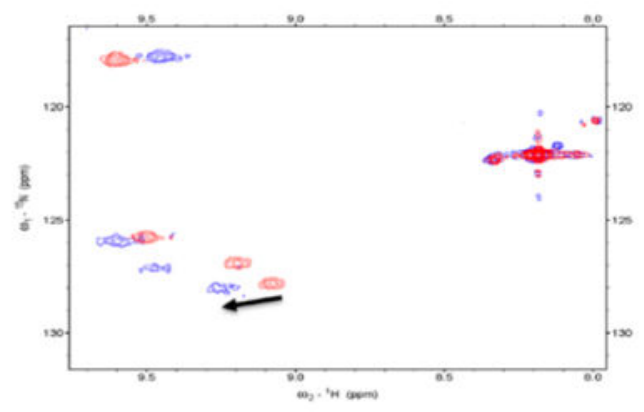

B

Figure 3.

Superposition of ${ }^{15} \mathrm{~N}-{ }^{1} \mathrm{H}$ HSQC spectra of (A) ${ }^{15} \mathrm{~N}$-Lys labeled and (B) ${ }^{15} \mathrm{~N}$-Phe labeled Robo1-Ig1-2, engineered with lanthanide binding peptide loaded with $\mathrm{Lu}^{3+}$ (red) or $\mathrm{Tm}^{3+}$ (blue). 


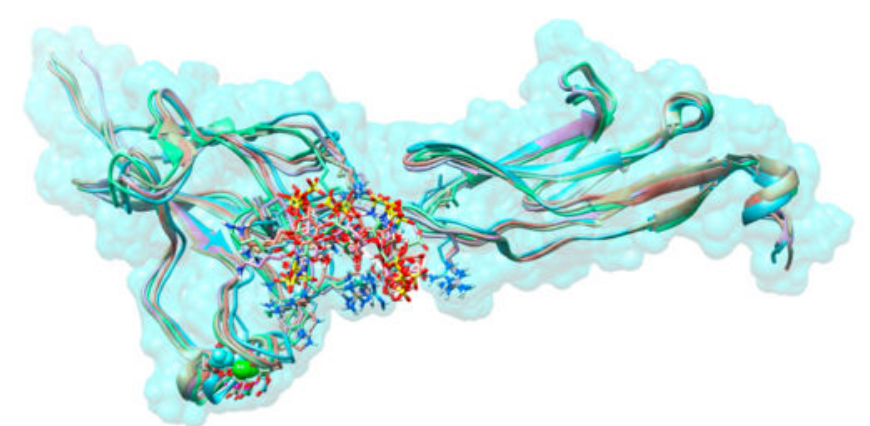

A

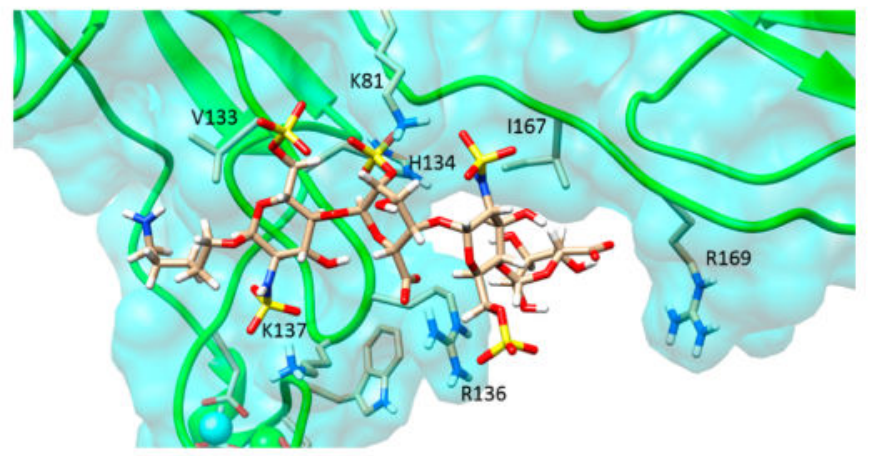

B

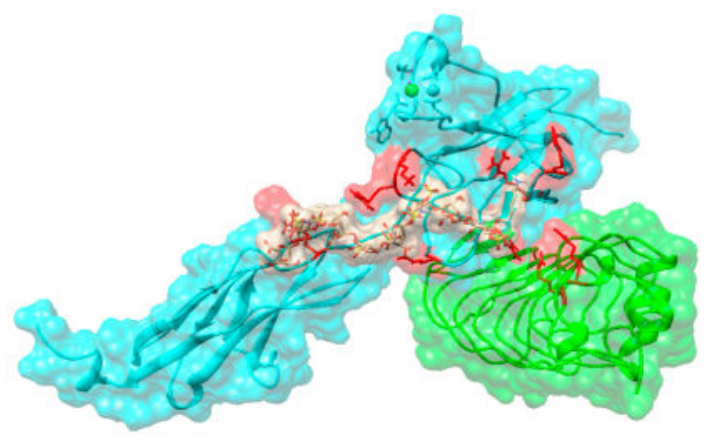

C

Figure 4.

(A) Top 5 HADDOCK structures of Robo1-Ig1-2-HS overlaid. (B) Expanded view of the binding pocket for the best HADDOCK structure. Interacting residues within $1 \AA$ of van der Waals contact are presented in a stick representation. (C) Model of trimeric Robo1-Ig1-2 (blue)-HS octamer (beige)-Slit (green) complex with positive residues labeled in red. 

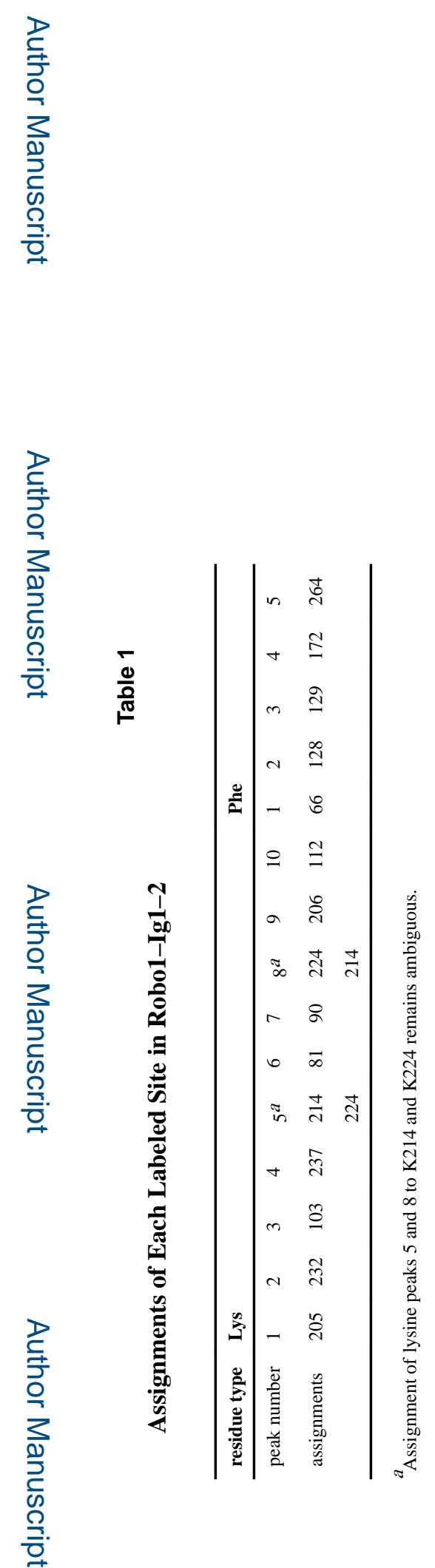

ACS Chem Biol. Author manuscript; available in PMC 2017 November 18. 


\section{롤 \\ ํㅗㄹ}

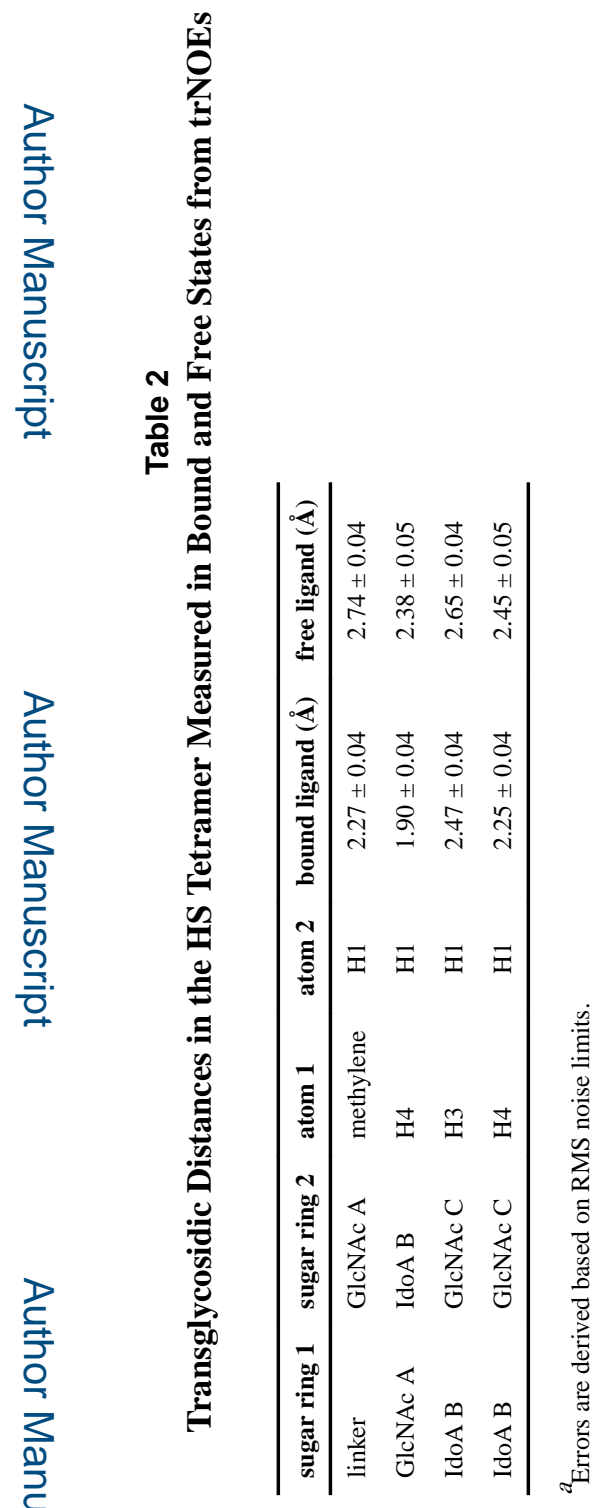

ACS Chem Biol. Author manuscript; available in PMC 2017 November 18. 

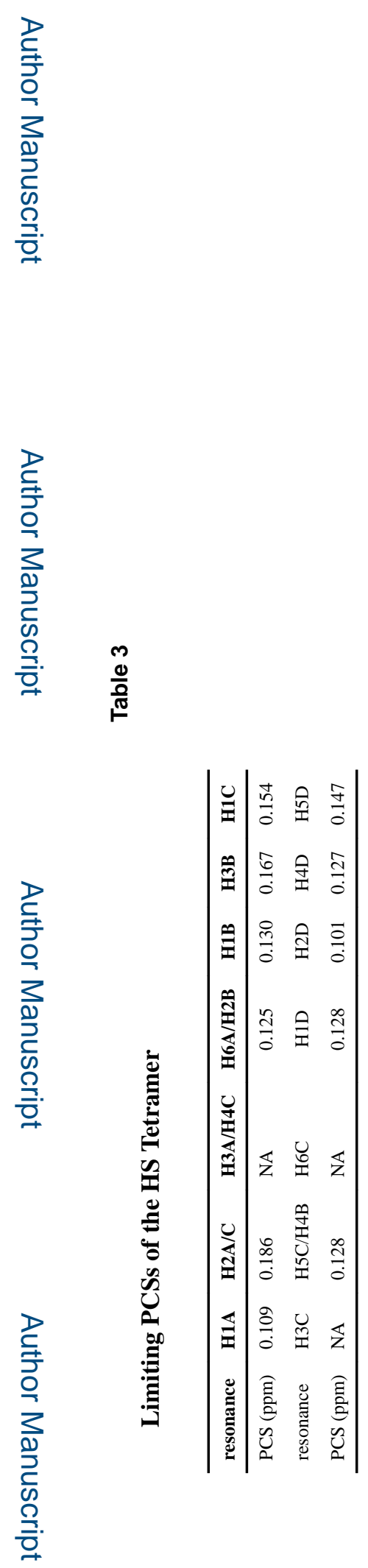

ACS Chem Biol. Author manuscript; available in PMC 2017 November 18. 
Table 4

Pairwise Electrostatic Interactions Involved in the Binding of the HS Tetramer to Robo1Ig1-2

\begin{tabular}{lllc}
\multicolumn{5}{c}{ Ig1-2 } & & \\
\hline & & & \\
\hline sugar residue & substituent & interacting amino acid & distance $(\stackrel{\AA}{)})$ \\
\hline GlcNAc A & N-sulfate & K137 & 2.0 \\
IdoA B & carboxylate & R136 & 1.6 \\
IdoA B & 2-O-sulfate & K81 & 1.8 \\
IdoA B & 2-O-sulfate & H134 & 2.9 \\
GlcNAc C & N-sulfate & K81 & 1.8 \\
GlcNAc C & 6-O-sulfate & R136 & 3.0 \\
IdoA D & carboxylate & R169 & 4.1 \\
\hline
\end{tabular}

